This is the postprint version of the following article: Martín A, Vázquez-Villoldo N, Gómez-Vallejo V, et al. In vivo imaging of system xc- as a novel approach to monitor multiple sclerosis. European Journal of Nuclear Medicine and Molecular Imaging. 2015;43(6):1124-1138 which has been published in final form at 10.1007/s00259-015-3275-3. This article may be used for non-commercial purposes in accordance with Springer Terms and Conditions for Self-Archiving. 


\title{
In vivo imaging of system xc- as a novel approach to monitor multiple sclerosis
}

Running head: Novel imaging approach to monitor MS

\author{
Abraham Martín $^{1 *}$, Nuria Vázquez-Villoldo ${ }^{2}, V$ anessa Gómez-Vallejo ${ }^{1,3}$, Daniel Padro ${ }^{1,4}$, Federico N. Soria ${ }^{2}$, \\ Boguslaw Szczupak ${ }^{1}$, Sandra Plaza-García ${ }^{1,4}$, Ander Arrieta ${ }^{1}$, Torsten Reese ${ }^{1,4}$, Jordi Llop ${ }^{1,3}$, Maria Domercq ${ }^{2}$, \\ Carlos Matute 2* $^{*}$ \\ ${ }^{1}$ Molecular Imaging Unit, CIC biomaGUNE, $\mathrm{P}^{\mathrm{o}}$ Miramon 182, San Sebastian, Spain. \\ ${ }^{2}$.Department of Neurosciences, University of the Basque Country, Barrio Sarriena s/n, 48940 Leioa, Spain, \\ Achucarro Basque Center for Neuroscience-UPV/EHU, 48170 Zamudio, Spain and Instituto de Salud Carlos III, \\ Centro de Investigación Biomédica en Red de Enfermedades Neurodegenerativas (CIBERNED), 48940 Leioa, \\ Spain. \\ ${ }^{3 .}$ Radiochemistry and Nuclear Imaging, CIC biomaGUNE, P ${ }^{\circ}$ Miramon 182, San Sebastian, Spain. \\ 4. Magnetic Resonance Imaging. CIC biomaGUNE, $\mathrm{P}^{\mathrm{o}}$ Miramon 182, San Sebastian, Spain. \\ (*both corresponding authors) \\ Corresponding authors:
}

Abraham Martín, Unidad de Imagen molecular; CICbiomaGUNE, Edificio Empresarial "C", Po Miramon 182, San Sebastian, Spain. E-mail address: amartin@cicbiomagune.es.

Carlos Matute, Department of Neurosciences, University of the Basque Country, Barrio Sarriena s/n, 48940 Leioa, Spain, Achucarro Basque Center for Neuroscience-UPV/EHU, 48170 Zamudio, Spain. E-mail address: carlos.matute@ehu.es.

Number of characters in title: 118, Number of characters in running head: 36 , Number of words in the abstract: 244, Number of words in the Introduction: 485, Number of words in the Discussion: 1330, Number of words in the body of the manuscript: 4884, Number of figures: 7

Keywords: $\mathrm{T}_{1} \mathrm{~W}-\mathrm{MRI} ;\left[{ }^{18} \mathrm{~F}\right] \mathrm{FDG} ;\left[{ }^{11} \mathrm{C}\right] \mathrm{PK} 11195 ;\left[{ }^{18} \mathrm{~F}\right] \mathrm{FSPG}$; PET; multiple sclerosis 


\begin{abstract}
Purpose Glutamate excitotoxicity contributes to oligodendroglial and axonal damage in multiple sclerosis pathology. Extracellular glutamate concentration in the brain is controlled by cystine/glutamate antiporter (xc- system), a membrane antiporter that imports cystine and releases glutamate. Despite this, the $\mathrm{xc}^{-}$system activity and its connection to the inflammatory reaction in multiple sclerosis (MS) is largely unknown.
\end{abstract}

Methods Longitudinal in vivo magnetic resonance (MRI) and positron emission tomography (PET) imaging studies with 2-[ $\left[{ }^{18} \mathrm{~F}\right]$ Fluoro-2-deoxy-D-glucose $\left(\left[{ }^{18} \mathrm{~F}\right] \mathrm{FDG}\right),\left[{ }^{11} \mathrm{C}\right]-(\mathrm{R})-(1-(2$-chlorophenyl)- $N$-methyl- $N$-1(1-methylpropyl)-3isoquinolinecarboxamide $\left(\left[{ }^{11} \mathrm{C}\right] \mathrm{PK} 11195\right)$ and $(4 \mathrm{~S})-4-\left(3-{ }^{18} \mathrm{~F}\right.$-fluoropropyl)-L-glutamate $\left(\left[{ }^{18} \mathrm{~F}\right] \mathrm{FSPG}\right)$ were carried out during the course of experimental autoimmune encephalomyelitis (EAE) induction in rats.

Results $\left[{ }^{18} \mathrm{~F}\right] \mathrm{FSPG}$ showed a significant increase of $\mathrm{xc}^{-}$system function in the lumbar section of the spinal cord at 14 days post immunization (dpi) that stands in agreement with the neurological symptoms and ventricle edema formation at this time point. Likewise, $\left[{ }^{18} \mathrm{~F}\right] \mathrm{FDG}$ did not show significant changes in glucose metabolism throughout central nervous system and $\left[{ }^{11} \mathrm{C}\right] \mathrm{PK} 11195$ evidenced a significant increase of microglial/macrophage activation in spinal cord and cerebellum two weeks after EAE induction. Therefore, $\left[{ }^{18}\right.$ F]FSPG showed a major capacity to discriminate regions of the central nervous system affected by the MS in comparison to $\left[{ }^{18} \mathrm{~F}\right] \mathrm{FDG}$ and $\left[{ }^{11} \mathrm{C}\right] \mathrm{PK} 11195$. Additionally, clodronate treated rats showed a depletion in microglial population and $\left[{ }^{18} \mathrm{~F}\right] \mathrm{FSPG}$ PET signal in spinal cord confirming a link between neuroinflammatory reaction and cystine/glutamate antiporter activity in EAE rats.

Conclusions Altogether, these results suggest that in vivo PET imaging of $\mathrm{xc}^{-}$system could become a valuable tool for the diagnosis and treatment evaluation of MS. 


\section{Introduction}

Multiple sclerosis (MS) is a chronic demyelinating disorder of the central nervous system (CNS) characterized by an autoimmune inflammatory reaction to myelin components leading to oligodendrocyte death, demyelination and axonal damage [1]. The clinical disease course usually starts with reversible episodes of neurological disability (relapsing-remitting MS, or RRMS), which later develops into a progressive stage with irreversible neurological decline (secondary progressive MS, or SPMS). Axonal loss is considered the main contributor to clinical disability [2] and occurs in both the acute and chronic phases of MS and its animal model, experimental autoimmune encephalomyelitis (EAE). Likewise, activated microglia and macrophages are thought to contribute to neurodegeneration, as their number correlates with the extent of axonal damage in MS lesions [3, 4]. One mechanism that could link activated microglia and macrophages to the formation of multiple sclerosis lesions could be the release of glutamate through the cystine/glutamate antiporter [5, 6]. Indeed, glutamate-mediated excitotoxicity is an important process in MS pathogenesis promoting oligodendroglial cell death and demyelization $[7,8]$. Neuroimaging techniques such as positron emission tomography (PET) have provided valuable information about the inflammatory component in MS, monitoring changes in regional glucose metabolism and the presence of activated microglia/macrophages in sclerotic plaques $[9,10]$. PET imaging of glucose metabolism with $\left[{ }^{18} \mathrm{~F}\right] \mathrm{FDG}$ has been applied to quantify inflammation due to the high metabolic rate of activated immune cells in EAE, the rat model for MS $[11,12]$ and in patients with MS [9]. Besides, radioligands that target the mitochondrial 18KDa translocator protein (TSPO) have been used for imaging microglia/monocytes in patients and animal models of MS $[10,13-17] .\left[{ }^{11} \mathrm{C}\right] \mathrm{PK} 11195$, the first TSPO radiotracer to be successfully applied in MS patients, has been able to detect the infiltration of monocytes in acute demyelinated lesions [18, 19]. Despite these advances, we still need new PET imaging techniques that correlate MS pathology with clinical disability in order to study disease progression and monitor the response to therapeutic drugs. In this regard, the evaluation of cystine/glutamate antiporter (xc ${ }^{-}$ system) following MS has been scarcely explored to date and might be potential candidate for PET imaging of MS.

(4S)-4-(3-(18)F-fluoropropyl)-l-glutamate ([ $\left.\left.{ }^{18} \mathrm{~F}\right] \mathrm{FSPG}\right)$ is a novel radiotracer to assess $\mathrm{xc}^{-}$transporter activity with PET which has been proposed as a promising radioligand presenting a wide range of diagnostic applications from cancer to neurologic diseases [20-22]. Therefore, the purpose of the present study was to investigate $\mathrm{xc}^{-}$transporter 
activity in rat central nervous system after EAE using PET with $\left[{ }^{18} \mathrm{~F}\right] \mathrm{FSPG}$. In particular, we were interested in investigating the relationship of $\mathrm{xc}^{-}$system with inflammatory reaction in EAE. For these reasons, rats were subjected to $\mathrm{T} 1$ weighted magnetic resonance imaging ( $\left.\mathrm{T}_{1} \mathrm{~W}-\mathrm{MRI}\right)$ and PET studies with $\left[{ }^{18} \mathrm{~F}\right] \mathrm{FDG}$ and $\left[{ }^{11} \mathrm{C}\right] \mathrm{PK} 11195$ following EAE. Likewise, $\left[{ }^{18} \mathrm{~F}\right] \mathrm{FSPG}$ binding was used to evaluate the therapeutic effect of clodronate, an inhibitor of microglia/macrophage. Therefore, the results reported here may contribute to develop new imaging-based strategies for monitoring disease progression, therapeutic responses and drug development in MS. 


\section{Materials and Methods}

\section{Induction of experimental autoimmune encephalomyelitis and treatment}

Adult male Lewis rats ( 8 week-old weighing 200-220g) $(n=21)$ were used. Animal studies were approved by the animal ethics committee of CIC biomaGUNE and local authorities and were conducted in accordance with the Directives of the European Union on animal ethics and welfare. EAE was induced by subcutaneous injection of inoculum containing $100 \mu \mathrm{g}$ of guinea pig myelin basic protein (Sigma) diluted in water, emulsified in equal volumes of Freund's incomplete adjuvant (Sigma) and supplemented with $500 \mu \mathrm{g}$ of heat-inactivated M. tuberculosis H37Ra (DIFCO Laboratories) in both hind feet. Eleven rats were repeatedly examined before (day 0) and at 7, 14, 21 and 28 after EAE induction to evaluate the temporal PET binding of glucose metabolism (n=6), TSPO $(n=6)$ and $\mathrm{xc}^{-}(\mathrm{n}=5)$. The animals studied at day 0 have been considered as the baseline control group. Likewise, four of these six rats were also subjected to MRI scans. Eight days after EAE induction, a group of ten rats were deeply anaesthetized with ketamine/xylazine and stereotactically injected in both lateral ventricles (AP $1.0 \mathrm{~mm} ; \mathrm{L} \pm 1.5 \mathrm{~mm}$; DV $-4.0 \mathrm{~mm}$ from skull) with a blunt Hamilton syringe loaded with $15 \mu \mathrm{L}$ of clodronatecontaining liposomes $(\mathrm{n}=5 ; 5 \mathrm{mg} / \mathrm{mL}$; ClodLip BV, Netherlands) or PBS-containing liposomes $(\mathrm{n}=5)$ at a flow rate of $1 \mu \mathrm{L} / \mathrm{min}$. Both suspensions were gently mixed by inversion immediately before loading the syringe. No mortality was observed after the clodronate or PBS injection. At day fourteen, treated and control rats were imaged with PET to determine the effect of clodronate on the $\mathrm{xc}^{-}$system function.

\section{Neurological evaluation}

The neurological impairment was monitored and scored daily on every animal from day 10 to day 28 post immunization according to the following scale ranging from 0 to 8 : 0 , normal; 1 , flaccid tail; 2 , tail paralysis; 3 , loss of muscle tone in hindlimbs; 4, hindlimbs hemiparalysis; 5, complete hindlimbs paralysis; 6, moderate paraparesis; 7, tetraparalysis; 8 , death. Total score could range from 0 (normal) to 8 (highest handicap) point-scale.

\section{Magnetic resonance imaging}


MRI scans were performed in rats $(\mathrm{n}=4)$ before (day 0$)$ and at 7, 14, 21 and 28 days after EAE induction. Before the scans, anesthesia was induced with $4 \%$ isoflurane and maintained by $2-2.5 \%$ of isofluorane in $100 \% \mathrm{O}_{2}$ during the scan. Animals were placed into a rat holder compatible with MRI acquisition systems and maintained normothermic using a water-based heating blanket at $37^{\circ} \mathrm{C}$. Measurements were performed by using an 11.7T Bruker Biospec system (Bruker, Ettlingen, Germany) with a $72 \mathrm{~mm}$ volumetric quadrature coil for excitation and a commercial $20 \mathrm{~mm}$ rat brain surface coil for reception. Acquisition parameters were as follows; Gradient echo sequence TR/TE $=500 / 2 \mathrm{~ms}$, fov $=30 \times 30 \mathrm{~mm}$, ACQ matrix $=256 \times 256$, slice thickness $=0.50 \mathrm{~mm}$, number of slices $=40$.

\section{Magnetic Resonance Imaging Image Analysis}

The MRI images were used to calculate the brain ventricle volume. Regions of interest were manually defined using the Open Source software 3D Slicer image analysis software (Version 3.6.3; www.slicer.org) for each rat on the region of brain ventricles. The total brain ventricle volume was calculated by summing the area of the ventricle region of all slices.

\section{Radiochemistry}

The production of $\left[{ }^{11} \mathrm{C}\right] \mathrm{PK} 11195$ was carried out by reaction of $\left[{ }^{11} \mathrm{C}_{\mathrm{C}} \mathrm{CH}_{3} \mathrm{I}\right.$ produced from $\left[{ }^{11} \mathrm{C}\right] \mathrm{CH}_{4}$ in a TRACERlab $\mathrm{FX}_{\mathrm{C}}$-Pro synthesis module (GE Healthcare, Waukesha, WI, USA) with N-Methyl-2-(2-amino-4cyanophenylthio)-benzylamine (MASB, $1 \mathrm{mg}$ in $80 \mu \mathrm{L}$ of dimethylsulfoxide, ABX, Radeberg, Germany) using the captive solvent method [23] (reaction time $=6 \mathrm{~min}, \mathrm{~T}=25^{\circ} \mathrm{C}$ ). Purification by high performance liquid chromatography (HPLC) and reformulation using solid phase extraction yielded the final solution of pure injectable tracer. The presence of the labeled species was confirmed by HPLC and co-elution with reference standard. Typical radiochemical yields (RCY) and specific activities (SA) were $33 \pm 5 \%$ (end of bombardment, EOB) and $135 \pm 18$ $\mathrm{GBq} / \mu \mathrm{mol}$ (end of synthesis), respectively (total synthesis time $=50 \mathrm{~min}$ ). Radiochemical purity was $>98 \%$.

The synthesis of (4S)-4-[3-[ $\left.{ }^{18} \mathrm{~F}\right]$ fluoropropyl]-L-glutamate $\left(\left[{ }^{18} \mathrm{~F}\right] \mathrm{FSPG}\right)$ was performed by ${ }^{18} \mathrm{~F}$-fluorination of the protected precursor di-tert-butyl (2S,4S)-2-tert-butoxycarbonylamino-4-(3-nitrophenylsulfonyloxy-propyl)pentanedioate, followed by acidic hydrolysis. The precursor preparation and the radiolabeling were carried out as 
previously described [24]. Radiochemical yields (EOB) were in the range 30-35\% and radiochemical purity was always $>93 \%$ at the time of injection.

$\left[{ }^{18}\right.$ F]-2-fluoro-2-deoxy-D-glucose $\left(\left[{ }^{18} \mathrm{~F}\right] \mathrm{FDG}\right)$ was kindly provided by IBA Molypharma (San Sebastian, Spain).

\section{Positron emission tomography scans and data acquisition}

PET scans were performed before (day 0) and at 7, 14, 21 and 28 days after EAE induction using a General Electric eXplore Vista CT camera (GE Healthcare). Scans were performed in rats anaesthetized with $4 \%$ isofluorane and maintained by $2-2.5 \%$ of isofluorane in $100 \% \mathrm{O}_{2}$. The tail vein was catheterized with a 24 -gauge catheter for intravenous administration of the radiotracer. Animals were placed into a rat holder compatible with PET acquisition system and maintained normothermic using a water-based heating blanket. (i) A group of six animals was subjected to two PET scans to assess TSPO binding $\left(\left[{ }^{11} \mathrm{C}\right] \mathrm{PK} 11195\right)$ and glucose metabolism $\left(\left[{ }^{18} \mathrm{~F}\right] \mathrm{FDG}\right)$ at every time point before and after EAE induction. Firstly, around $30 \mathrm{MBq}$ of $\left[{ }^{11} \mathrm{C}\right] \mathrm{PK} 11195$ were injected concomitantly with the start of the PET acquisition. Whole body dynamic images (28 frames: 4x2, 4x5, 4x10, 4x15, 4x30, 4x60, 4x120 seconds) were acquired in 4 bed positions in the $400-700 \mathrm{keV}$ energetic window, with a total acquisition time of 64.5 minutes, providing a $175 \times 175$ matrix with a pixel size of $0.887 \mathrm{~mm}$ and 220 slices. Secondly, after at least 180 minutes (approximately, 9 half-lives of ${ }^{11} \mathrm{C}$ ), animals received an intravenous bolus injection of $\left[{ }^{18} \mathrm{~F}\right] \mathrm{FDG}$ (around $30 \mathrm{MBq}$ ). Before the injection, animals were fasted for 4 hours and after a $\left[{ }^{18} \mathrm{~F}\right] \mathrm{FDG}$ uptake period of 45 minutes, the animals were re-anesthetized and placed on the PET for a whole body static scan acquired for a total of 60 minutes in 4 bed positions (15 minutes per bed). (ii) A second group of fifteen animals was subjected to a PET scan to assess $\mathrm{xc}^{-}$ system binding $\left(\left[{ }^{18} \mathrm{~F}\right] \mathrm{FSPG}\right)$ before and after EAE induction and to evaluate the effect of the treatment with clodronate. Around $30 \mathrm{MBq}$ of $\left[{ }^{18} \mathrm{~F}\right] \mathrm{FSPG}$ were injected concomitantly with the start of the PET acquisition. Whole body dynamic images (17 frames: $2 \times 2,2 \times 5,4 \times 10,4 \times 30,3 \times 60,2 \times 300$ seconds) with a total acquisition of 63.6 minutes were acquired in 4 bed positions following the same protocol than that used for $\left[{ }^{11} \mathrm{C}\right] \mathrm{PK} 11195$. After each PET scan, CT acquisitions were also performed $(140 \mu \mathrm{A}$ intensity, $40 \mathrm{kV}$ voltage), providing anatomical information of each animal as well as the attenuation map for the later image reconstruction. Dynamic acquisitions were 
reconstructed (decay and CT-based attenuation corrected) with 2DOSEM using 4 iterations/16 subsets. CT was reconstructed with FeldKamp method with binning 2.

\section{Positron emission tomography image analysis}

PET images were analyzed using PMOD image analysis software version 3.506 (PMOD Technologies Ltd, Zürich, Switzerland). To verify the anatomical location of the signal, PET images were co-registered to the anatomical data of the CT of the same animal. Five types of Volumes of Interest (VOIs) were established along the central nervous system. Two VOIs were generated in the brain (cerebrum and the cerebellum) and three VOIs in the spinal cord (cervical, thoracical and lumbar) to evaluate $\left[{ }^{11} \mathrm{C}\right] \mathrm{PK} 11195,\left[{ }^{18} \mathrm{~F}\right] \mathrm{FDG}$ and $\left[{ }^{18} \mathrm{~F}\right] \mathrm{FSPG}$ PET signal over time. VOIs were manually drawn in the inner part of the selected regions using the rat CT image for each PET scan. Therefore, the reduced size of the VOI in the spinal cord in regard to the resolution of the PET scanner may lead to a partial volume effect that could affect the PET binding on this particular region. For static $\left[{ }^{18} \mathrm{~F}\right] \mathrm{FDG}$ PET scans quantification, the mean value and the standard deviation (mean \pm sd) of each VOI corresponding to the total uptake for 60 minutes were expressed as percentage of injected dose per cubic centimeter (\%ID/cc). For dynamic PET studies, last time frames in steady state were used to calculate the summed PET binding uptake during the last 16 and 20 minutes of acquisition for $\left[{ }^{11} \mathrm{C}\right] \mathrm{PK} 11195$ and $\left[{ }^{18} \mathrm{~F}\right] \mathrm{FSPG}$, respectively. Finally, PET signal frames were summed and voxels outside the selected VOIs were masked to better represent the PET binding in the central nervous system using PMOD software.

\section{Immunohistochemistry}

To check microglial depletion, 7 days after clodronate injection (15 days after EAE induction) rats were anaesthetized with chloral hydrate $(200 \mathrm{mg} / \mathrm{ml}, 0.5 \mathrm{~g} / \mathrm{kg})$ and perfused with $0.1 \mathrm{M}$ phosphate buffer (PB) followed by paraformaldehyde (PFA) 4\% in $0.1 \mathrm{M}$ PB. Free-floating spinal cord vibratome sections $(50 \mu \mathrm{m})$ were incubated in blocking-permeabilization solution containing $0.1 \%$ Triton-X100 and 5\% NGS in PBS for $1 \mathrm{~h}$ at room temperature (RT). Slices were subsequently incubated with the rabbit anti-Iba1 (Wako, 1:200) overnight at $4^{\circ} \mathrm{C}$. After 3 washing steps in PBS, tissue was incubated with goat anti-rabbit Alexa Fluor ${ }^{\circledR} 488$ (1:200; Molecular Probes) for 1 h at RT in darkness. Slices were stained with Hoechst for nuclear identification and imaged using an Olympus Fluoview 
FV500 confocal microscope. For quantification of Iba1 staining, Z-stacks (step size $=0.5 \mu \mathrm{m}$ ) were obtained in spinal cord (lumbar portion, longitudinal sections) of at least 3 slices per animal. The area stained was calculated with ImageJ software (NIH, USA) using an automatic default threshold applied on the maximal projection of each stack, to sample all possible planes and avoid bias by antibody penetration.

\section{Statistical analyses}

For PET signal values, the statistical analysis was performed as follows; $\% \mathrm{ID} / \mathrm{cc}$ for each animal, brain region (cerebrum and cerebellum) and spinal cord (cervical, thoracic and lumbar) were calculated at each time point. $\% \mathrm{ID} / \mathrm{cc}$ values within each region and time point were averaged and compared with the averaged baseline control values (before EAE induction) using one-way ANOVA followed by Dunnet's multiple-comparison tests for post hoc analysis. The effect of clodronate in EAE rats was compared with control EAE rats using an unpaired $t$ test. The level of significance was regularly set at $\mathrm{p}<0.05$. Statistical analyses were performed with GraphPad Prism version 4.02 software.

\section{RESULTS}

\section{In vivo imaging experimental set-up based on clinical outcome of EAE}

The clinical severity of EAE in Lewis rats was monitored from day 10 to day 28 after immunization. All immunized rats developed typical clinical signs of acute EAE. The first clinical signs occurred over day 10 after EAE induction with both tail paralysis and loss of muscle tone in hindlimbs. Subsequently, disease progressed from hemiparalysis at days 12 to 13 to complete hindlimbs paralysis at day 14 post-immunization. EAE symptoms persisted for about 7 days followed by a progressive recovery from day 21 to day 28 after EAE induction (Figure 1). To determine whether brain ventricular volume (MRI), glucose metabolism $\left(\left[{ }^{18} \mathrm{~F}[\mathrm{FDG})\right.\right.$, TSPO $\left(\left[{ }^{11} \mathrm{C}\right] \mathrm{PK} 11195\right)$ and $\mathrm{xc}^{-}$system $\left(\left[{ }^{18} \mathrm{~F}\right] \mathrm{FSPG}\right)$ expression changes can correlate with clinical symptoms or pathologic damage by EAE, rats were serially imaged by both MRI and PET/CT at (i) day 0 as naïve control, (ii) day 7 (before the paralysis), (iii) day 14 
as the peak of clinical severity of EAE, (iv) day 21 as clinical remission and (v) day 28 as recovery of the disease. Likewise, the efficacy of the treatment with clodronate was evaluated by PET with $\left[{ }^{18}\right.$ F]FSPG at day 14 after immunization (Figure 1).

\section{Ventricle volume evolution after EAE}

We applied MRI- $\mathrm{T}_{1} \mathrm{~W}$ imaging to investigate the pathological manifestations after EAE induction. Images showed ventricle volume evolution at control (day 0) and 7, 14, 21 and 28 days after EAE induction (Figure 2A-E). Rat ventricle volume increased significantly from circa $13 \mathrm{~mm}^{3}$ at day 0 to ca. $25 \mathrm{~mm}^{3}$ at day 14 and followed by a progressive decrease to reach control values at day 28 after immunization $(P<0.05$, Figure $2 \mathrm{~F})$. These results evidenced that the highest brain ventricle volume following EAE run in parallel with the peak of clinical severity of the disease in rats.

\section{PET Imaging of glucose metabolism in EAE-induced rats}

Next, we studied the glucose metabolism using $\left[{ }^{18} \mathrm{~F}\right] \mathrm{FDG}$ in brain (cerebrum, cerebellum) and spinal cord after EAE induction (Figure 3, $\mathrm{n}=6,1$ rat died between days 14-21). The images with normalized color scale illustrate the evolution of the PET signals in the central nervous system (CNS) at control (day 0) and days 14 and 28 after EAE induction (Figure 3A-C). PET images evidenced a higher $\left[{ }^{18} \mathrm{~F}\right] \mathrm{FDG}$ binding uptake in the brain than in the spinal cord at different time points before and after EAE induction (Figure 3A-C). In the whole brain, the binding values for $\left[{ }^{18}\right.$ F]FDG presented a slight decrease at 7 days post immunization and followed by a non-significant increase of the PET signal from day 14 to day 28 after EAE induction compared to control (day 0) values (Figure 3D-E). Likewise, the glucose metabolism of the cervical spinal cord over time showed similar values than that observed before immunization (Figure 3F). In the thoracic spinal cord, $\left[{ }^{18} \mathrm{~F}\right] \mathrm{FDG}$ evidenced a non-significant hypometabolism over the following month post immunization (Figure 3G). These results stand in agreement with the reduction of the glucose metabolic rate in patients with multiple sclerosis $[25,26]$. In contrast, lumbar spinal card showed similar

$\left[{ }^{18}\right.$ F]FDG binding values at different time points after EAE induction (Figure $3 \mathrm{H}$ ). Therefore, these results did not 
demonstrate the existence of a correlation between the consumption of glucose with the progression of the disease over time.

\section{TSPO imaging with [ $\left.{ }^{11} \mathrm{C}\right]$ PK11195 PET after EAE induction}

The time course of TSPO was evaluated using $\left[{ }^{11} \mathrm{C}\right] \mathrm{PK} 11195$ in brain (cerebrum, cerebellum) and spinal cord (cervical, thoracic and lumbar) after EAE induction (Figure 4, n=6, 1 rat died between days 14-21). Representative images of whole body scans showed the $\left[{ }^{11} \mathrm{C}\right] \mathrm{PK} 11195$ binding distribution in the CNS at 0,14 and 28 days after immunization (Figure 4A-C). The intact or non-inflamed cerebral tissue shows a very low TSPO receptor expression followed by a dramatic up-regulation due to the inflammatory reaction evidencing it usefulness to image neuroinflammatory processes [27]. This fact has been supported by the very low $\left[{ }^{11} \mathrm{C}\right] \mathrm{PK} 11195$ binding level observed at the regions evaluated before (day 0) the EAE induction. Likewise, all studied regions in brain and spinal cord have exhibited a similar TSPO over-expression following EAE (Figure 4). The cerebrum was the region of the central nervous system that experienced the lowest $\left[{ }^{11} \mathrm{C}\right] \mathrm{PK} 11195$ increase following EAE induction. At day 14, a non-significant increase of TSPO expression with respect to control (day 0) was followed by a subsequent decrease from days 21 to 28 post immunization (Figure 4D). In contrast, the cerebellum showed a significant increase of the $\left[{ }^{11} \mathrm{C}\right] \mathrm{PK} 11195$ PET signal at day 14 followed by a progressive decrease later on $(P<0.05$ with respect to control animals, Figure 4E). In the spinal cord, $\left[{ }^{11} \mathrm{C}\right] \mathrm{PK} 11195$ displayed a progressive increase from day 0 , peaking at day 14 and followed by a progressive signal decline from day 21 to day 28 after EAE induction $(P<0.05$ with respect to control animals, Figure 4F-H). Altogether, these results evidenced a global increase of the inflammatory reaction and TSPO over-expression in the spinal cord and cerebellum that stand in agreement with both the clinical features and the ventricle volume increase of acute EAE in rats.

\section{PET $x^{-}$binding uptake in CNS after EAE}

Cystine/glutamate antiporter system function was evaluated with $\left[{ }^{18} \mathrm{~F}\right] \mathrm{FSPG}$ in brain (cerebrum and cerebellum) and spinal cord (cervical, thoracic and lumbar) after EAE induction (Figure 5, $\mathrm{n}=5$ ). Representative images illustrate the evolution of the $\left[{ }^{18}\right.$ F]FSPG PET signals in the CNS before and at days 14 and 28 post-immunization (Figure 5A-C). 
Studied regions in the brain exhibited similar control $\left[{ }^{18} \mathrm{~F}\right] \mathrm{FSPG}$ binding values at different time points following EAE induction in rats (Figure 5D,E). Spinal cord evidenced a higher xc- expression at control (day 0) conditions in comparison to that in brain (Figure 5F-H). At day 7, both cervical and thoracic spinal cord sections showed a nonsignificant $\left[{ }^{18} \mathrm{~F}\right] \mathrm{FSPG}$ binding decrease and a recovery from days 14 to 28 post immnunization restoring control values (Figure 5F,G). In contrast, lumbar spinal cord showed a significant over-increase of the $\left[{ }^{18} \mathrm{~F}\right] \mathrm{FSPG}$ PET signal at day 14 followed by a progressive decrease from day 21 to day 28 after EAE induction $(P<0.05$ with respect to control animals; Figure 5H). Hence, $\mathrm{xc}^{-}$system up-regulation was exhibited in the lumbar region which might result in higher glutamate release and excitotoxic damage leading demyelination in this specific section of the spinal cord [6].

\section{Microglial regulation of $x c^{-}$system expression with $\left[{ }^{18}\right.$ F]FSPG after EAE}

$\mathrm{xc}^{-}$system upregulation could be related to neuroinflammation, as previously described [6]. To determine whether microglial cells are responsible of system $\mathrm{xc}^{-}$activity upregulation in EAE, we selectively depleted these cells by intracerebroventricular injection of liposome-encapsulated clodronate after EAE induction [28]. Eight days post immunization (dpi), rats were treated either with clodronate $(n=5)$ or vehicle $(n=5)$. The rats were subjected to

$\left[{ }^{18}\right.$ F]FSPG PET and immunofluorescence studies at days 6 (14 dpi, peak of EAE symptoms) and 7 (15 dpi) posttreatment (Figure 6A). Treated EAE rats with clodronate tended to increase the severity of neurological symptoms after EAE induction when compared to vehicle injected rats (Figure 6B). As expected, clodronate liposomes reduced significantly the number of $\mathrm{Iba1}^{+}$microglial cells in hippocampal CA1 region and spinal cord $(P<0.05$ with respect to control EAE animals; Figure $6 \mathrm{C}, \mathrm{D})$. PET imaging with $\left[{ }^{18} \mathrm{~F}\right] \mathrm{FSPG}$ at day 6 after treatmet with clodronate showed a decrease of the binding uptake in relation to control EAE rats (Figure 7A, B). Treatment with clodronate showed a non-significant PET signal decrease in cervical and thoracic sections of the spinal cord (Figure 7C,D). In contrast, clodronate induced a significant reduction of $\left[{ }^{18} \mathrm{~F}\right] \mathrm{FSPG}$ binding in relation to non-treated EAE rats in lumbar section of the spinal cord $\left(P<0.05\right.$, Figure 7E), suggesting the contribution of microglia on $\mathrm{xc}^{-}$system activity after EAE. 


\section{DISCUSSION}

The diagnosis and progression of MS might be supported by molecular imaging techniques when clinical symptoms have not been conclusive. Despite MRI has been used to detect the spatiotemporal distribution of sclerotic lesions [29], this technique has shown several disadvantages as lack of specificity to detect the pathologic features of MS [30]. Likewise, PET has been applied to monitor specific biological aspects of MS as glucose metabolism [11, 12], activated microglia/macrophages [14, 16, 17, 31] demyelination and remyelination [32-34] in rodent models of EAE. Nevertheless, clinical studies using PET have been mainly focused on the evaluation of changes in glucose metabolism and activated microglia/macrophages to evaluate the progression and therapy efficacy of MS [19, 25, 26]. Despite these findings, several pathological processes of interest for MS evolution remain still unexplored by PET imaging. Hence, the identification of new up-regulated transporters in reactive glial cells as cystine/glutamate antiporter $\mathrm{xc}^{-}$that promotes the release of extracellular glutamate and excitotoxic damage to oligodendrocytes [6] is of great interest to the in vivo imaging research of MS. Because of this, we have assessed in parallel the in vivo expression of $\mathrm{xc}^{-}$system, TSPO and glucose metabolism in reactive glial cells using PET imaging procedures in combination with MRI, immunohistochemistry and neurofunctional evaluation after EAE in rats longitudinally.

\section{Neurological outcome and ventricle dilatation after EAE}

The EAE in Lewis rats is characterized by a period of relapse and remission, thus reproducing hallmarks of the disease $[16,17]$. Immunized rats showed clinical signs over day 10 after EAE induction followed by a peak of symptoms at day 14 post immunization with complete hindlimb paralysis. The symptoms persisted for about 7 days and followed by recovery to almost normal motor function skills. Hence, the clinical evolution of the disease was the rational for the design of the experimental set-up with both MRI and PET/CT in the present study (Figure 1). Multiple sclerosis is a chronic disease of the central nervous system characterized by inflammatory processes leading brain edema and myelin damage [35]. In the present study, in vivo MRI-T $\mathrm{T}_{1} \mathrm{~W}$ showed a significant increase of the cerebral ventricle volume at day 14 followed by a swelling decrease from days 21 to 28 after EAE induction (Figure 2). The increase of ventricular enlargement runs in parallel with the peak of MS symptoms at day 14 and 
followed by a progressive improvement at days 21-28 after immunization (Figure 1). Likewise, these results were consistent with ventricle volume changes in a relapsing-remitting model of EAE in mice [35].

\section{PET imaging of glucose metabolism with $I^{18}$ F]FDG}

Previous in vivo PET studies in MS patients have been used as a marker of disease activity [25] evidencing decrease in glucose metabolism as a result of severe cognitive and mental dysfunction underlying MS [26, 36]. In contrast, $\left[{ }^{18}\right.$ F]FDG has also been used to image neuroinflammation and to detect inflammatory infiltrates in spinal cord of the EAE model in rats [12] and mice[11]. Despite this, Buck et al., also demonstrated that $\left[{ }^{18}\right.$ F]FDG presented a low sensitivity in detecting inflammatory infiltrates because of the high background $\left[{ }^{18} \mathrm{~F}\right] \mathrm{FDG}$ signal in the CNS [12]. In the present study, no significant differences in brain and spinal cord $\left[{ }^{18} \mathrm{~F}\right] \mathrm{FDG}$ uptake were detected that stand in agreement with the findings showed by de Paula Faria and collaborators in a EAE rat model [31] (Figure 3). In fact, the lack of changes in $\left[{ }^{18} \mathrm{~F}\right] \mathrm{FDG}$ uptake observed in our study might be due to the increase of $\left[{ }^{18} \mathrm{~F}\right] \mathrm{FDG}$ signal as consequence of inflammatory response that is masked by a reduction of metabolism in degenerated CNS regions.

\section{In vivo PET imaging TSPO after EAE}

TSPO receptors have become a potential target for the development of radiotracers to study neuroinflammation with PET due to its over-expression in response to the inflammatory environment [37]. [ $\left.{ }^{11} \mathrm{C}\right] \mathrm{PK} 11195$ is the first TSPO radioligand that could detect microglia/macrophages activation in MS patients $[18,19]$ and in rodents after EAE induction $[18,35]$. Likewise, novel radiotracers have been recently used for imaging microglial activation in spinal cords of EAE $[16,17]$. The present study showed an overall TSPO expression increase in cerebellum and spinal cord at day 14 after EAE induction that run in parallel with the neurological severity and ventricle enlargement reached at this time point (Figure 4). The fact that the highest $\left[{ }^{11} \mathrm{C}\right] \mathrm{PK} 11195$ binding uptake at control conditions was found in the thoracic region of the spinal cord might be attributed to spill-over effects from the lungs which have a high expression of TSPO [38]. Besides, this is the reason for the lack of statistically PET binding differences in the thoracic spinal cord in comparison to cervical and lumbar sections. The highest $\left[{ }^{11} \mathrm{C}\right] \mathrm{PK} 11195$ binding uptake was observed in the lumbar spinal cord and progressively decreased from caudal to rostral sections of the CNS as 
follows; thoracic cord $>$ cervical cord $>$ cerebellum $>$ cerebrum. These findings are consistent with Xie and collaborators who showed a similar ranking order for TSPO binding in spinal cords of EAE in Lewis rats [16]. Therefore, our results evidence an ascending inflammatory response that is most severe in the caudal regions of the spinal cord and decreases towards rostral regions of the CNS.

\section{PET imaging of $x c^{-}$system in CNS after EAE}

Previously, in vivo brain imaging of multiple sclerosis patients with magnetic resonance spectroscopy at 3T (MRS) showed increased glutamate concentrations in acute lesions and normal-appearing white matter [39]. Despite these findings, the intercellular and subcellular compartmentalization of the neurotransmitter pool cannot be distinguished by MRS. Hence, we took advance of the recently developed radioligand $\left[{ }^{18}\right.$ F]FSPG for imaging cystine/glutamate antiporter (system $\mathrm{xc}^{-}$) activity by PET [20-22]. This transport system is a sodium-independent transporter that mediates the cellular uptake of cystine in exchange for glutamate at a 1:1 ratio [21]. Likewise, this transporter is considered the main source of non-synaptic glutamate that contributes to enhance neuronal damage in ischemia and glioma-derived epileptic seizures $[22,40]$. System $\mathrm{xc}^{-}$is not able to discriminate between cystine and glutamate for the inward transport [41] and the $\left[{ }^{18}\right.$ F]FSPG radioligand, analog of glutamate, internalizes into the cell displacing cystine, allowing to measure $\mathrm{xc}^{-}$functional activity. Therefore, this is the first study to evaluate the activity of the cystine/glutamate antiporter (system $\mathrm{xc}^{-}$) with $\left[{ }^{18} \mathrm{~F}\right] \mathrm{FSPG}$ PET in a EAE rat model. Previously, $\left[{ }^{18} \mathrm{~F}\right] \mathrm{FSPG}$ has shown promising diagnostic properties with a high cancer detection rate in patients with lung and liver cancer [20, 21]. Likewise, $\left[{ }^{18} \mathrm{~F}\right] \mathrm{FSPG}$ uptake showed a significant increase of cystine/glutamate expression and function at few minutes and hours after acute cerebral ischemia in rats [22]. Surprisingly, $\left[{ }^{18} \mathrm{~F}\right] \mathrm{FSPG}$ displayed a different binding profile than that showed by $\left[{ }^{11} \mathrm{C}\right] \mathrm{PK} 11195$, a lack of PET binding uptake in the cerebellum and a significant PET signal increase in only the lumbar section of the spinal cord at day 14 post immunization (Figure 5). Thus, the difference between $\left[{ }^{11} \mathrm{C}\right] \mathrm{PK} 11195$ and $\left[{ }^{18} \mathrm{~F}\right] \mathrm{FSPG}$ might be due to the existence of different microglial/macrophage populations throughout the CNS following MS and only activated microglia at lumbar spinal cord contribute to glutamate release and oligodendroglial damage, as revealed by $\left[{ }^{18} \mathrm{~F}\right] \mathrm{FSPG}$ PET imaging. Besides, taking into account that EAE symptoms progress up from caudal to rostral section of the spinal cord, our results evidence the potential of $\left[{ }^{18} \mathrm{~F}\right] \mathrm{FSPG}$ (i) to evaluate the spatiotemporal progression of the EAE in rats and (ii) to discriminate 
spinal cord regions according to the damage degree following experimental MS. Likewise, these results also show the increase of the cystine/glutamate antiporter function in accordance with the complete hindlimb paralysis of EAE rats.

\section{Ex vivo and in vivo evidences of microglial regulation of $x c^{-}$system activity after $E A E$}

To confirm the relationship between the system $x^{-}$activity and neuroinflammation, microglia were selectively depleted with intracerebroventricular injection of liposome-encapsulated clodronate after EAE induction [32].EAE rats treated with clodronate evidenced a significant decrease on Ibal positive cells in the CA1 region of the hippocampus and in the spinal cord at day 15 after EAE induction (Figure 6). Despite the microglial depletion, treated EAE rats with clodronate did not show a decrease on neurological symptoms at day 14 after immunization. In contrast, Popovich and collaborators observed hindlimb recovery after depletion of hematogenous macrophages by chronic intravenous clodronate treatment in EAE rats [42]. Hence, the absence of clinical recovery in our study might point towards hematogenous macrophages as a cause of the clinical severity in EAE [43]. Despite these differences, $\left[{ }^{18}\right.$ F]FSPG PET showed a significant binding decrease in the lumbar section of the spinal cord in those rats treated with clodronate in comparison to control EAE rats (Figure 7), evidencing the microglial source of cystine/glutamate antiporter activity after EAE in rats.

\section{Summary and conclusions}

We report here the PET imaging of $\left[{ }^{18}\right] \mathrm{FSPG}$ to assess cystine/glutamate antiporter (xc ${ }^{-}$system) bioavailability and its relationship with neuroinflammatory response after EAE in rats. These results confirmed a binding increase of $\left[{ }^{18} \mathrm{~F}\right] \mathrm{FSPG}$ in the lumbar spinal cord at the second week after EAE which is consistent with the clinical severity evolution and the ventricle dilatation after experimental MS in rats. Likewise, $\left[{ }^{18}\right]$ FSPG showed to be more selective and suitable radioligand than $\left[{ }^{11} \mathrm{C}\right] \mathrm{PK} 11195$ and $\left[{ }^{18} \mathrm{~F}\right] \mathrm{FDG}$ for the diagnosis and evaluation of EAE. In addition, treatment with clodronate decreased microglial population and $\left[{ }^{18} \mathrm{~F}\right] \mathrm{FSPG}$ PET binding, evidencing the connection between inflammation and cystine/glutamate antiporter activity in EAE rats. Finally, these findings propose a novel 
imaging approach to image multiple sclerosis that might open new avenues into the diagnosis and therapy evaluation of this disease.

\section{Acknowledgements}

The authors would like to thank M González, A Leukona and M Errasti for technical support in the radiosynthesis, A Cano and C Muñoz for technical assistance in the PET studies.

\section{Compliance with ethical standards}

Funding: This study was funded by MINECO SAF2010-21547 and SAF2013-45084-R (C. M) and SAF2014-54070JIN (A.M), FEDER, Department of Industry of Basque Government (IE14-385) and CIBERNED.

Conflict of interests: The authors declare no competing financial interests.

Ethical approval: Animal studies were approved by the animal ethics committee of CIC biomaGUNE and local authorities and were conducted in accordance with the Directives of the European Union on animal ethics and welfare.

\section{References}

1. H. Lassmann, J. van Horssen, D. Mahad. Progressive multiple sclerosis: pathology and pathogenesis. Nat Rev Neurol. 2012;8:647-656.

2. S.S. Zamvil, L. Steinman. Diverse targets for intervention during inflammatory and neurodegenerative phases of multiple sclerosis. Neuron. 2003;38:685-688.

3. A. Bitsch, T. Kuhlmann, C. Da Costa, S. Bunkowski, T. Polak, W. Bruck. Tumour necrosis factor alpha mRNA expression in early multiple sclerosis lesions: correlation with demyelinating activity and oligodendrocyte pathology. Glia. 2000;2:366-375. 
4. S. Rasmussen, Y. Wang, P. Kivisakk, R.T. Bronson, M. Meyer, J. Imitola, et al. Persistent activation of microglia is associated with neuronal dysfunction of callosal projecting pathways and multiple sclerosis-like lesions in relapsing--remitting experimental autoimmune encephalomyelitis. Brain. 2007;130:2816-2829.

5. M. Domercq, M.V. Sanchez-Gomez, C. Sherwin, E. Etxebarria, R. Fern, C. Matute. System xc- and glutamate transporter inhibition mediates microglial toxicity to oligodendrocytes. J Immunol. 2007;178:6549-6556.

6. O. Pampliega, M. Domercq, F.N. Soria, P. Villoslada, A. Rodriguez-Antiguedad, et al. Increased expression of cystine/glutamate antiporter in multiple sclerosis. J Neuroinflammation. 2011;8:1742-2094.

7. C. Matute, M.V. Sanchez-Gomez, L. Martinez-Millan, R. Miledi. Glutamate receptor-mediated toxicity in optic nerve oligodendrocytes. Proc Natl Acad Sci U S A, 1997;94:8830-8835.

8. M. Domercq, E. Etxebarria, A. Perez-Samartin, C. Matute. Excitotoxic oligodendrocyte death and axonal damage induced by glutamate transporter inhibition. Glia. 2005;52:36-46.

9. C. Schiepers, P. Van Hecke, R. Vandenberghe, S. Van Oostende, P. Dupont, P. Demaerel, et al. Positron emission tomography, magnetic resonance imaging and proton NMR spectroscopy of white matter in multiple sclerosis. Mult Scler. 1997;3:8-17.

10. J.C. Debruyne, J. Versijpt, K.J. Van Laere, F. De Vos, J. Keppens, K. Strijckmans, et al. PET visualization of microglia in multiple sclerosis patients using [11C]PK11195. Eur J Neurol. 2003;10:257-264.

11. C.G. Radu, C.J. Shu, S.M. Shelly, M.E. Phelps, O.N. Witte. Positron emission tomography with computed tomography imaging of neuroinflammation in experimental autoimmune encephalomyelitis. Proc Natl Acad Sci U S A. $2007 ; 104: 1937-1942$.

12. D. Buck, A. Forschler, C. Lapa, T. Schuster, P. Vollmar, T. Korn, et al. 18F-FDG PET detects inflammatory infiltrates in spinal cord experimental autoimmune encephalomyelitis lesions. J Nucl Med. 2012;53:1269-1276.

13. A. Takano, F. Piehl, J. Hillert, A. Varrone, S. Nag, B. Gulyas, et al. In vivo TSPO imaging in patients with multiple sclerosis: a brain PET study with [18F]FEDAA1106. EJNMMI Res. 2013;3:3-30.

14. L. Airas, A.M. Dickens, P. Elo, P. Marjamaki, J. Johansson, O. Eskola, et al. In vivo PET imaging demonstrates diminished microglial activation after fingolimod treatment in an animal model of multiple sclerosis. J Nucl Med. 2015;56:305-310 
15. F. Mattner, M. Staykova, P. Berghofer, H.J. Wong, S. Fordham, P. Callaghan, et al. Central nervous system expression and PET imaging of the translocator protein in relapsing-remitting experimental autoimmune encephalomyelitis. J Nucl Med. 2013;54:291-298.

16. L. Xie, T. Yamasaki, N. Ichimaru, J. Yui, K. Kawamura, K. Kumata, et al. [(11)C]DAC-PET for noninvasively monitoring neuroinflammation and immunosuppressive therapy efficacy in rat experimental autoimmune encephalomyelitis model. J Neuroimmune Pharmacol. 2012;7:231-242.

17. G. Abourbeh, B. Theze, R. Maroy, A. Dubois, V. Brulon, Y. Fontyn, et al. Imaging microglial/macrophage activation in spinal cords of experimental autoimmune encephalomyelitis rats by positron emission tomography using the mitochondrial $18 \mathrm{kDa}$ translocator protein radioligand [(1)(8)F]DPA-714. J Neurosci. 2012;32:5728-5736. 18. E. Vowinckel, D. Reutens, B. Becher, G. Verge, A. Evans, T. Owenset al. PK11195 binding to the peripheral benzodiazepine receptor as a marker of microglia activation in multiple sclerosis and experimental autoimmune encephalomyelitis. J Neurosci Res. 1997;50:345-353.

19. R.B. Banati, J. Newcombe, R.N. Gunn, A. Cagnin, F. Turkheimer, F. Heppner, et al. The peripheral benzodiazepine binding site in the brain in multiple sclerosis: quantitative in vivo imaging of microglia as a measure of disease activity. Brain. 2000;123:2321-2337.

20. S. Baek, C.M. Choi, S.H. Ahn, J.W. Lee, G. Gong, J.S. Ryu, et al. Exploratory clinical trial of (4S)-4-(3[18F]fluoropropyl)-L-glutamate for imaging xc- transporter using positron emission tomography in patients with non-small cell lung or breast cancer. Clin Cancer Res. 2012;18:5427-5437.

21. S. Baek, A. Mueller, Y.S. Lim, H.C. Lee, Y.J. Lee, G. Gong, et al. (4S)-4-(3-18F-fluoropropyl)-L-glutamate for imaging of $\mathrm{xC}$ transporter activity in hepatocellular carcinoma using PET: preclinical and exploratory clinical studies. J Nucl Med. 2013;54:117-123.

22. F.N. Soria, A. Perez-Samartin, A. Martin, K.B. Gona, J. Llop, B. Szczupak, Extrasynaptic glutamate release through cystine/glutamate antiporter contributes to ischemic damage. J Clin Invest. 2014;124:3645-3655.

23. A.A. Wilson, A. Garcia, L. Jin, S. Houle. Radiotracer synthesis from [(11)C]-iodomethane: a remarkably simple captive solvent method. Nucl Med Bio. 2000;1 27:529-532.

24. N. Koglin, A. Mueller, M. Berndt, H. Schmitt-Willich, L. Toschi, A.W. Stephens, et al. Specific PET imaging of xC- transporter activity using a (1)(8)F-labeled glutamate derivative reveals a dominant pathway in tumor metabolism. Clin Cancer Res. 2011;17:6000-6011. 
25. R. Bakshi, R.S. Miletich, P.R. Kinkel, M.L. Emmet, W.R. Kinkel. High-resolution fluorodeoxyglucose positron emission tomography shows both global and regional cerebral hypometabolism in multiple sclerosis. J Neuroimaging. 1998;8:228-234.

26. M. Blinkenberg, C.V. Jensen, S. Holm, O.B. Paulson, P.S. Sorensen. A longitudinal study of cerebral glucose metabolism, MRI, and disability in patients with MS. Neurology. 1999;53:149-153.

27. M.K. Chen, T.R. Guilarte. Translocator protein $18 \mathrm{kDa}$ (TSPO): molecular sensor of brain injury and repair. Pharmacol Ther. 2008;118:1-17.

28. J.V. Faustino, X. Wang, C.E. Johnson, A. Klibanov, N. Derugin, M.F. Wendland, et al. Microglial cells contribute to endogenous brain defenses after acute neonatal focal stroke. J Neurosci. 2011;31: 12992-13001.

29. W.I. McDonald, A. Compston, G. Edan, D. Goodkin, H.P. Hartung, F.D. Lublin, et al. Recommended diagnostic criteria for multiple sclerosis: guidelines from the International Panel on the diagnosis of multiple sclerosis. Ann Neurol.2001;50:121-127.

30. M. Filippi, M.A. Rocca, N. De Stefano, C. Enzinger, E. Fisher, M.A. Horsfield, et al. Magnetic resonance techniques in multiple sclerosis: the present and the future. Arch Neurol. 2011;68:1514-1520.

31. D. de Paula Faria, E.F. de Vries, J.W. Sijbesma, C.A. Buchpiguel, R.A. Dierckx, S.C. Copray. PET imaging of glucose metabolism, neuroinflammation and demyelination in the lysolecithin rat model for multiple sclerosis. Mult Scler. 2014;20:1443-1452.

32. B. Stankoff, Y. Wang, M. Bottlaender, M.S. Aigrot, F. Dolle, C. Wu, et al. Imaging of CNS myelin by positronemission tomography. Proc Natl Acad Sci U S A. 2006;103:9304-9309.

33. D. de Paula Faria, E.F. de Vries, J.W. Sijbesma, R.A. Dierckx, C.A. Buchpiguel, S. Copray. PET imaging of demyelination and remyelination in the cuprizone mouse model for multiple sclerosis: a comparison between [11C]CIC and [11C]MeDAS. Neuroimage. 2014;87:395-402.

34. D. de Paula Faria, M.L. Vlaming, S.C. Copray, F. Tielen, H.J. Anthonijsz, J.W. Sijbesma, et al. PET Imaging of Disease Progression and Treatment Effects in the Experimental Autoimmune Encephalomyelitis Rat Model. J Nucl Med. 2014;55:1330-1335.

35. R. Aharoni, E. Sasson, T. Blumenfeld-Katzir, R. Eilam, M. Sela, Y. Assaf, et al. Magnetic resonance imaging characterization of different experimental autoimmune encephalomyelitis models and the therapeutic effect of glatiramer acetate. Exp Neurol. 2013;240:130-144. 
36. M. Blinkenberg, K. Rune, C.V. Jensen, M. Ravnborg, S. Kyllingsbaek, S. Holm, et al. Cortical cerebral metabolism correlates with MRI lesion load and cognitive dysfunction in MS. Neurology. 2000;54:558-564.

37. A. Winkeler, R. Boisgard, A. Martin, B. Tavitian. Radioisotopic imaging of neuroinflammation. J Nucl Med. 2010;51:1-4.

38. R.B. Banati, R.J. Middleton, R. Chan, C.R. Hatty, W.W. Kam, C. Quin, et al. Positron emission tomography and functional characterization of a complete PBR/TSPO knockout. Nat Commun. 2014;5:5452.

39. R. Srinivasan, N. Sailasuta, R. Hurd, S. Nelson, D. Pelletier. Evidence of elevated glutamate in multiple sclerosis using magnetic resonance spectroscopy at 3 T. Brain. 2005;128:1016-1025.

40. S.C. Buckingham, S.L. Campbell, B.R. Haas, V. Montana, S. Robel, T. Ogunrinu, et al. Glutamate release by primary brain tumors induces epileptic activity. Nat Med. 2001;17:1269-1274.

41. S.A. Patel, B.A. Warren, J.F. Rhoderick, R.J. Bridges. Differentiation of substrate and non-substrate inhibitors of transport system xc(-): an obligate exchanger of L-glutamate and L-cystine. Neuropharmacology. 2004;46:273-284.

42. P.G. Popovich, Z. Guan, P. Wei, I. Huitinga, N. van Rooijen, B.T. Stokes. Depletion of hematogenous macrophages promotes partial hindlimb recovery and neuroanatomical repair after experimental spinal cord injury. Exp Neurol. 1999;158:351-365.

43. B. Ajami, J.L. Bennett, C. Krieger, K.M. McNagny, F.M. Rossi. Infiltrating monocytes trigger EAE progression, but do not contribute to the resident microglia pool. Nat Neurosci. 2011;14:1142-1149.

\section{Figure legends}

\section{Figure 1. EAE course and imaging.}

Time course of symptoms and time points for PET/CT and MRI studies before and after EAE induction. Typical neurological scores (mean \pm s.d.) for Lewis rats immunized with MBP/CFA at day $0(n=11)$ that peak at day 14 post immunization and regress completely from days 21-28 after EAE induction. Neurological scoring: 0, no symptoms; 1, flaccid tail; 2, tail paralysis; 3, loss of muscle tone in hind limbs; 4, hindlimbs hemiparalysis; 5, complete hindlimbs paralysis, 6 , moderate paraparesis; 7 , tetraparalysis; and 8 , death. Rats $(n=11)$ were serially imaged by 
MRI and PET/CT before (day 0) and at 7, 14, 21 and 28 dpi (grey bars). A group of rats were treated ("treatment arrow in the figure") with clodronate $(n=5)$ or vehicle $(n=5)$ eight days after EAE induction, and monitored with $\mathrm{PET} / \mathrm{CT}$ at day 14.

Figure 2. MRI imaging of structural changes during EAE course.

Serial representative coronal $\mathrm{T}_{1} \mathrm{~W}$-MRI images ranging from anterior-posterior axis before (day 0$)$ and at $7,14,21$ and 28 dpi (A-E). Images correspond to the same representative animal for each time. Ventricle brain volumes were repeteadly quantified in four rats just before immunization (day 0 ) and at 14 and 28 days after $\mathrm{EAE}(\mathrm{F}) .{ }^{*} P<0.05$ compared with control.

\section{Figure 3. $\left[{ }^{18}\right.$ F]FDG PET imaging during EAE course.}

Normalized sagittal PET images of $\left[{ }^{18} \mathrm{~F}\right] \mathrm{FDG}$ at day 0 (control) (A), day 14 (B) and day 28 (C) after EAE are coregistered with the $\mathrm{CT}$ of each animal to localize anatomically the PET signal. Images correspond to the same representative animal for each time point. The percentage of injected dose per cubic centimeter of $\left[{ }^{18} \mathrm{~F}\right] \mathrm{FDG}(\%$ $\mathrm{ID} / \mathrm{cc}$, mean \pm s.d.) was quantified in cerebrum (D), cerebellum (E), cervical (F), thoracic (G) and lumbar spinal cord $(\mathrm{H})$. The upper right panels of each figure show the selected ROIs for the quantification defined in a representation of the rat central nervous system. Rats ( $\mathrm{n}=6,1$ rat died between days 14-21) were repeatedly examined by PET before (day0) and at 7, 14, 21 and 28 days after EAE.

\section{Figure 4. $\left[{ }^{11} \mathrm{C}\right]$ PK11195 PET imaging during EAE course.}

Normalized sagittal PET images of $\left[{ }^{11} \mathrm{C}\right] \mathrm{PK} 11195$ at day 0 (control) (A), day 14 (B) and day 28 (C) after EAE are co-registered with the CT of each animal to localize anatomically the PET signal. Images correspond to the same

representative animal for each time point. The percentage of injected dose per cubic centimeter of $\left[{ }^{11} \mathrm{C}\right] \mathrm{PK} 11195(\%$ $\mathrm{ID} / \mathrm{cc}$, mean \pm s.d.) was quantified in cerebrum (D), cerebellum (E), cervical (F), thoracic (G) and lumbar spinal 
cord $(\mathrm{H})$. The upper right panels of each figure show the selected ROIs for the quantification defined in a representation of the rat central nervous system. Rats $(n=6,1$ rat died between days 14-21) were repeatedly examined by PET before (day0) and at 7, 14, 21 and 28 days after EAE. ${ }^{*} P<0.05$ compared with control.

Figure 5. $\left[{ }^{18}\right.$ F]FSPG PET imaging during EAE course.

Normalized sagittal PET images of $\left[{ }^{18}\right.$ F]FSPG at day 0 (control) (A), day 14 (B) and day 28 (C) after EAE are coregistered with the CT of each animal to localize anatomically the PET signal. Images correspond to the same representative animal for each time point. The percentage of injected dose per cubic centimeter of $\left[{ }^{18} \mathrm{~F}\right] \mathrm{FSPG}(\%$ ID/cc, mean \pm s.d.) was quantified in cerebrum (D), cerebellum (E), cervical (F), thoracic $(\mathrm{G})$ and lumbar spinal cord $(\mathrm{H})$. The upper right panels of each figure show the selected ROIs for the quantification defined in a representation of the rat central nervous system. Rats $(\mathrm{n}=5)$ were repeatedly examined by PET before (day 0$)$ and at 7, 14, 21 and 28 days after EAE. $* P<0.05$ compared with control.

Figure 6. Histochemical analysis of microglia depletion with clodronate in rats with EAE.

Immunofluorescence (IF) reveals depletion of microglial cells after treatment with clodronate in EAE rats. Clodronate effect over microglia and $\mathrm{xc}^{-}$system was evaluated with in vivo PET and ex vivo IF after EAE induction (A). Neurological scores for clodronate $($ Clod; $n=5)$ and vehicle $(P B S)(n=5)$ treated rats were evaluated at day 14 after EAE induction (B). Percentage of $\mathrm{Iba}^{+}{ }^{+}$microglial staining in CA1 region of hippocampus and in spinal cord of treated rats with clodronate and PBS (C). Immunofluorescent labeling of Iba1 (green) and Hoechst (blue) shows microglial and nuclear distribution in hippocampus and spinal cord in EAE rats treated with PBS and clodronate (D). ${ }^{*} P<0.05$ compared with control.

Figure 7. $\left[{ }^{18}\right.$ F]FSPG PET imaging during EAE course after clodronate treatment. 
Normalized sagittal PET images of $\left[{ }^{18}\right.$ F]FSPG for vehicle (A) and clodronate-treated (B) rats at day 14 after EAE induction are co-registered with the CT of each animal to localize anatomically the PET signal. Images correspond to the same representative animal for each time point. The percentage of injected dose per cubic centimeter of $\left[{ }^{18} \mathrm{~F}\right] \mathrm{FSPG}(\% \mathrm{ID} / \mathrm{cc}$, mean \pm s.d.) was quantified in cervical (C), thoracic (D) and lumbar spinal cord (E) in vehicle $(n=5)$ and clodronate-treated $(n=5)$ EAE rats. The upper right panels of each figure show the selected ROIs for the quantification defined in a representation of the rat spinal cord. ${ }^{*} P<0.05$ compared with control. 
Figure 1.

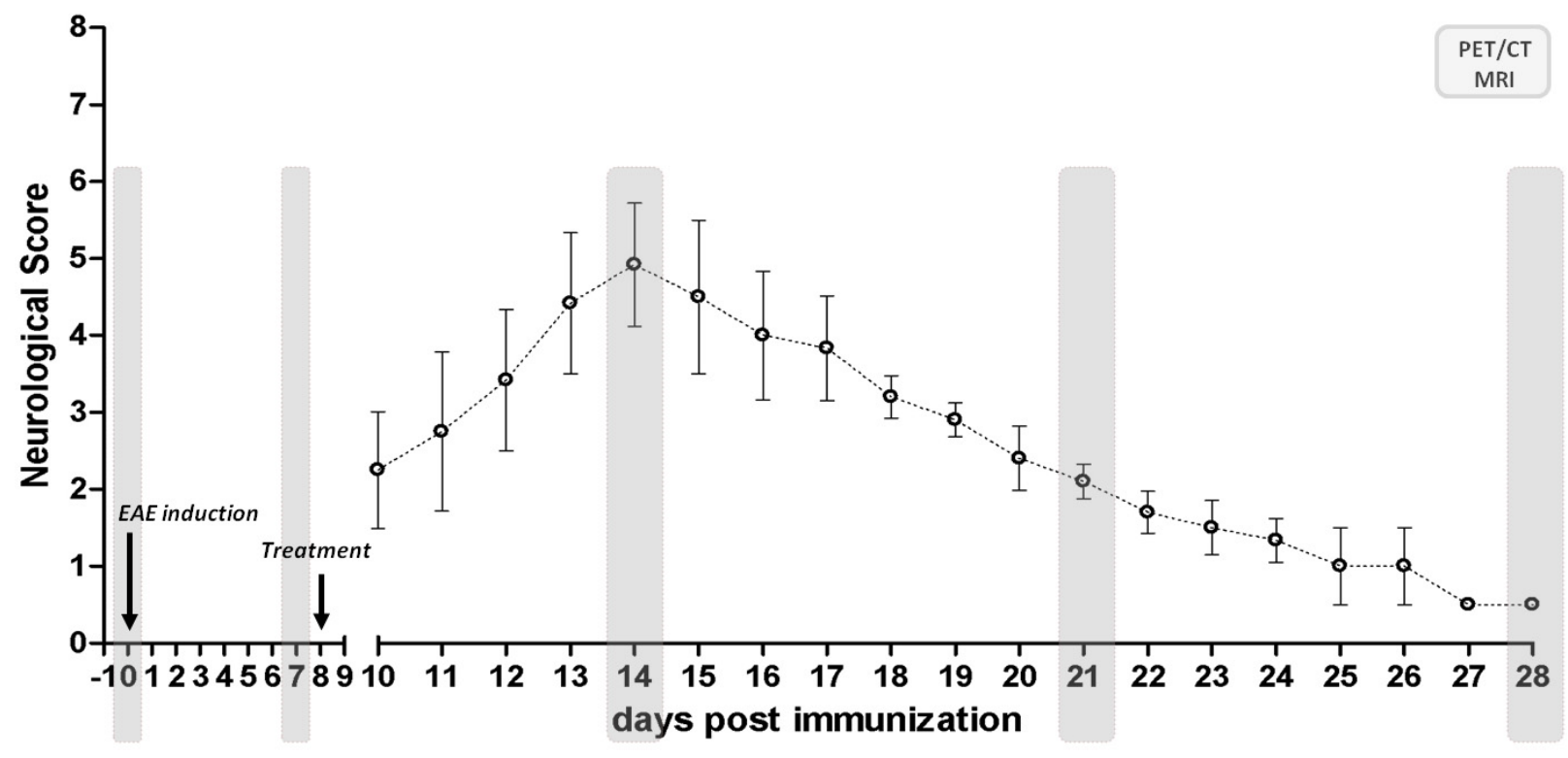


Figure 2.

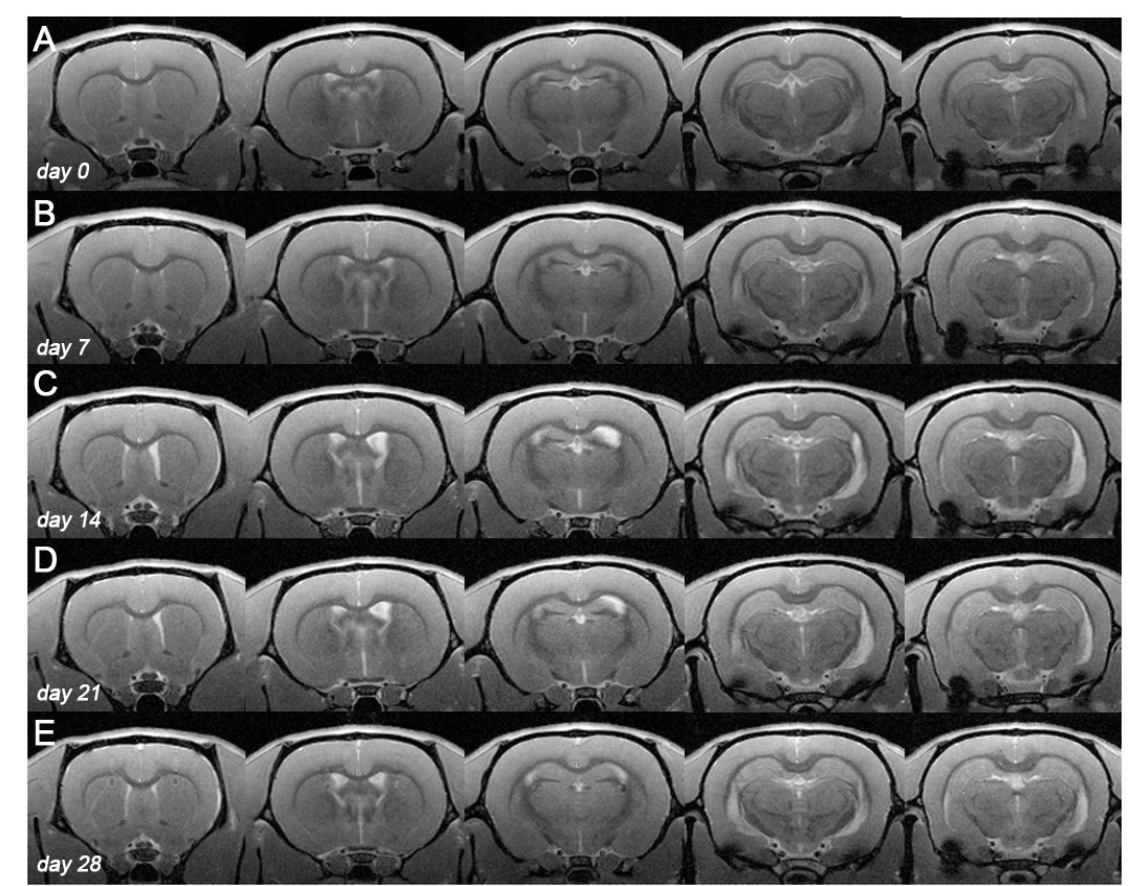

F

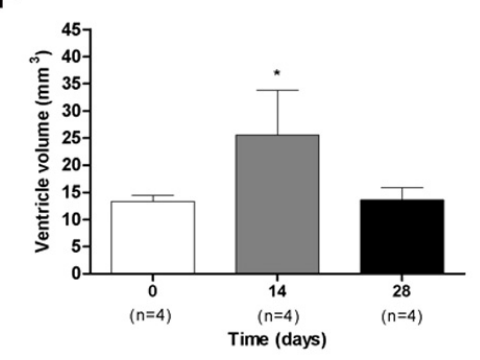


Figure 3.
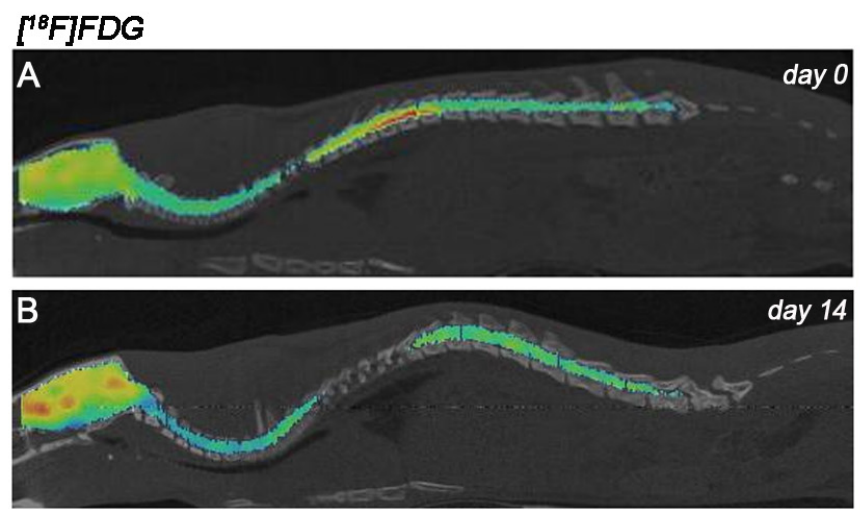

\%ID/cc

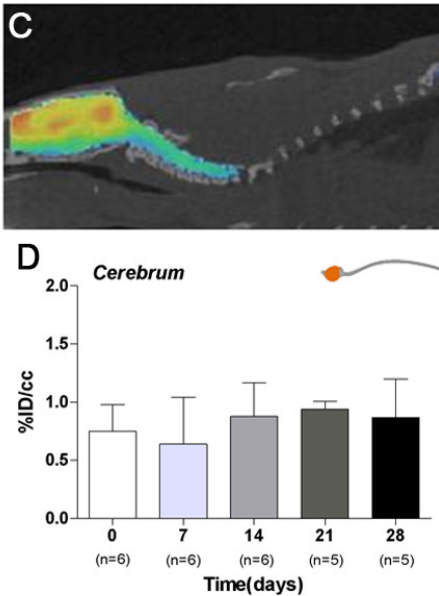

day 28

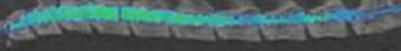

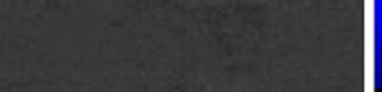

$E_{2.0}$ Cerebellum

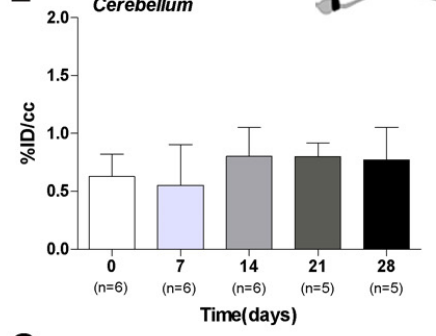

$\mathrm{F}$

G
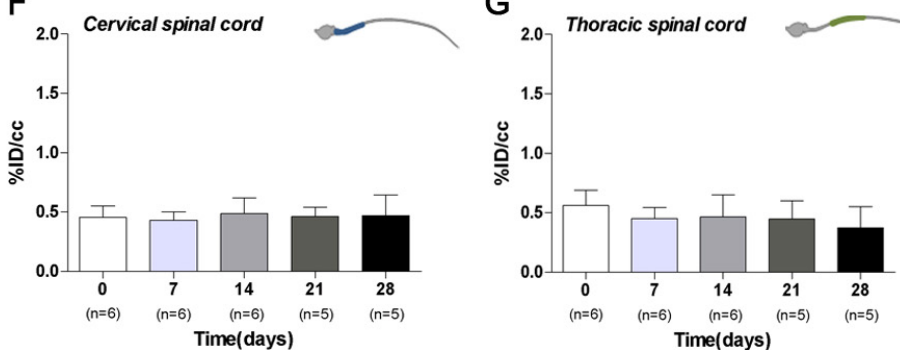

$\mathrm{H}$

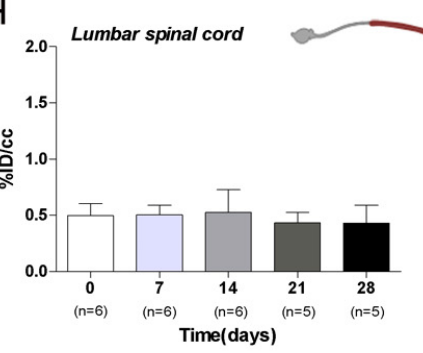


Figure 4.

["C]PK11195

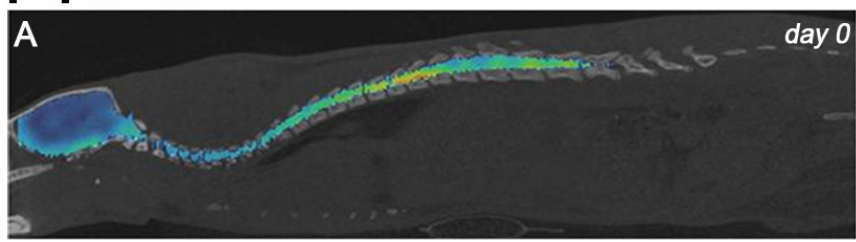

1.5

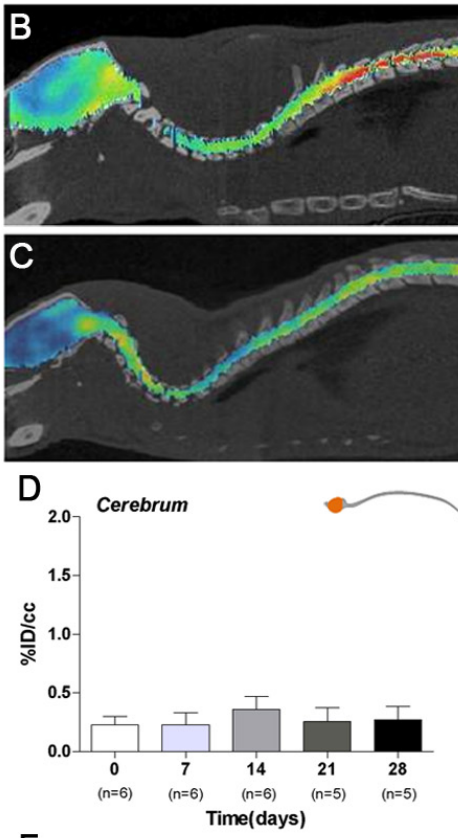

day 14
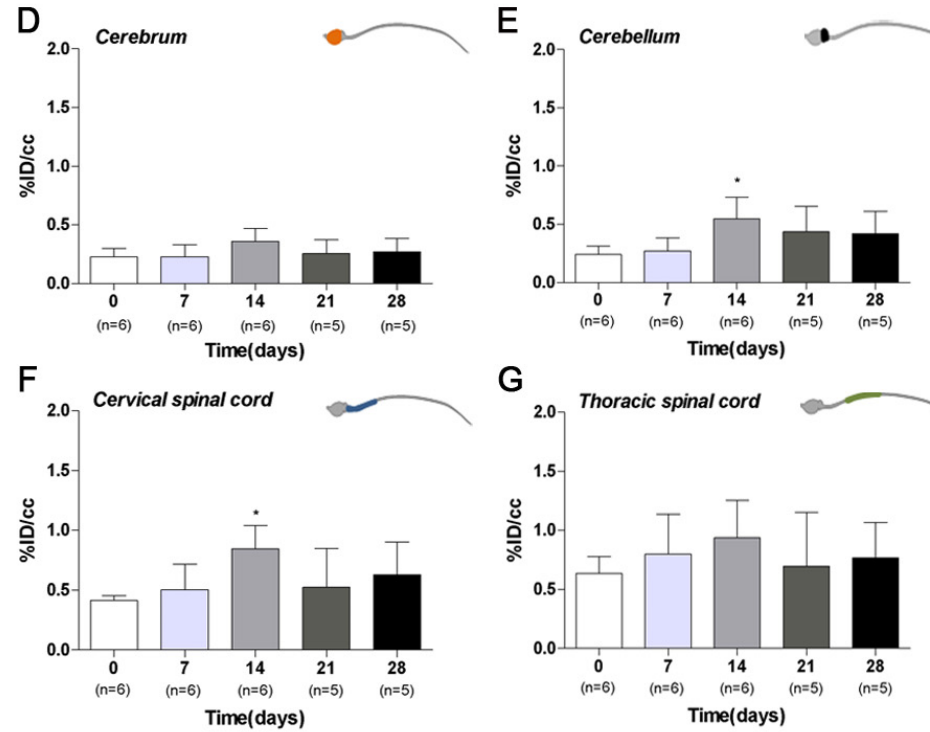

G
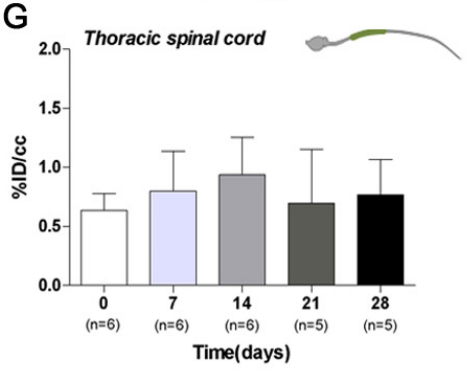

$\mathrm{H}$

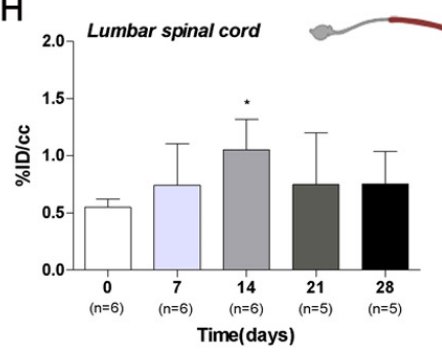




\section{Figure 5.}

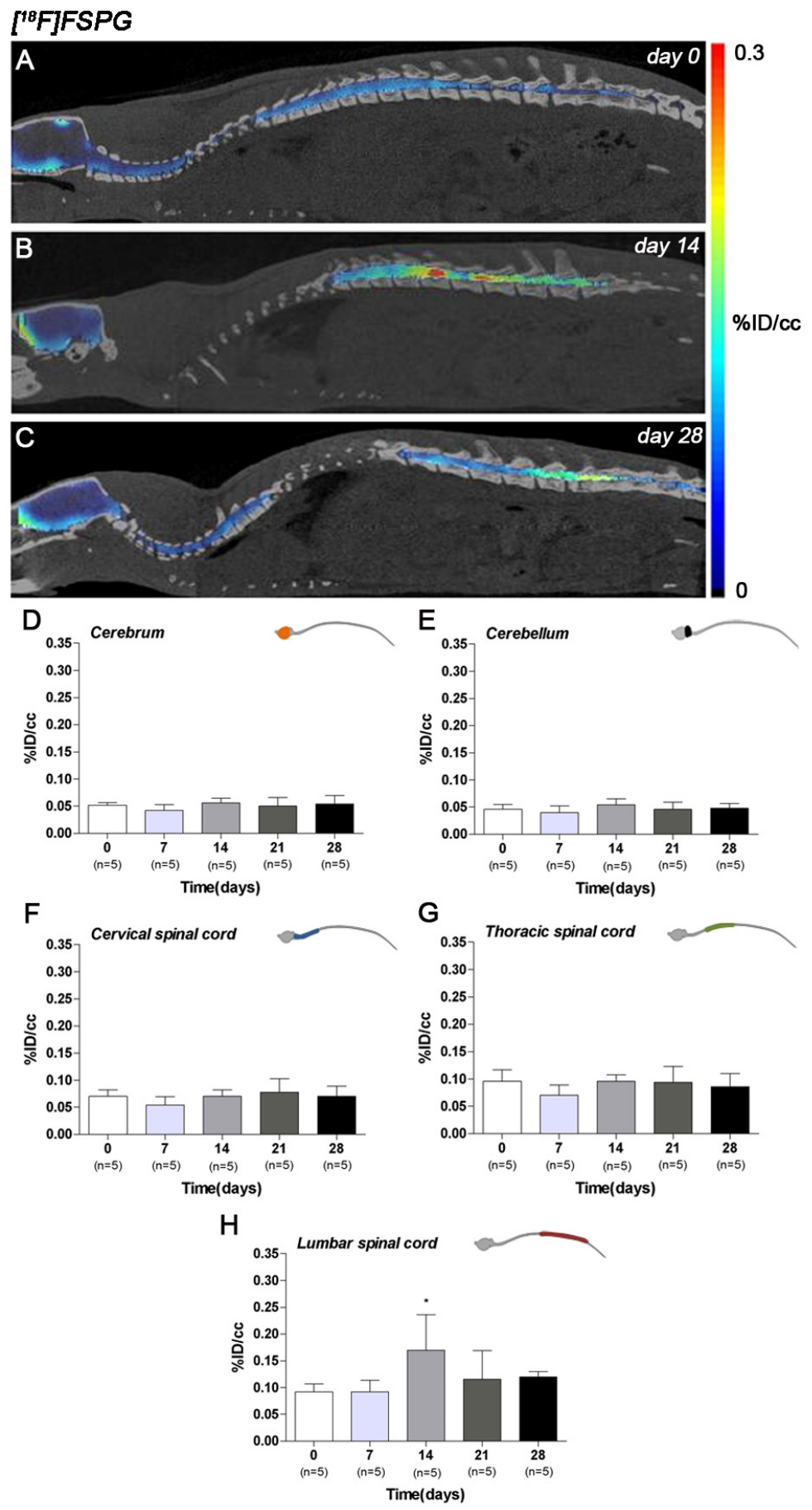


Figure 6.

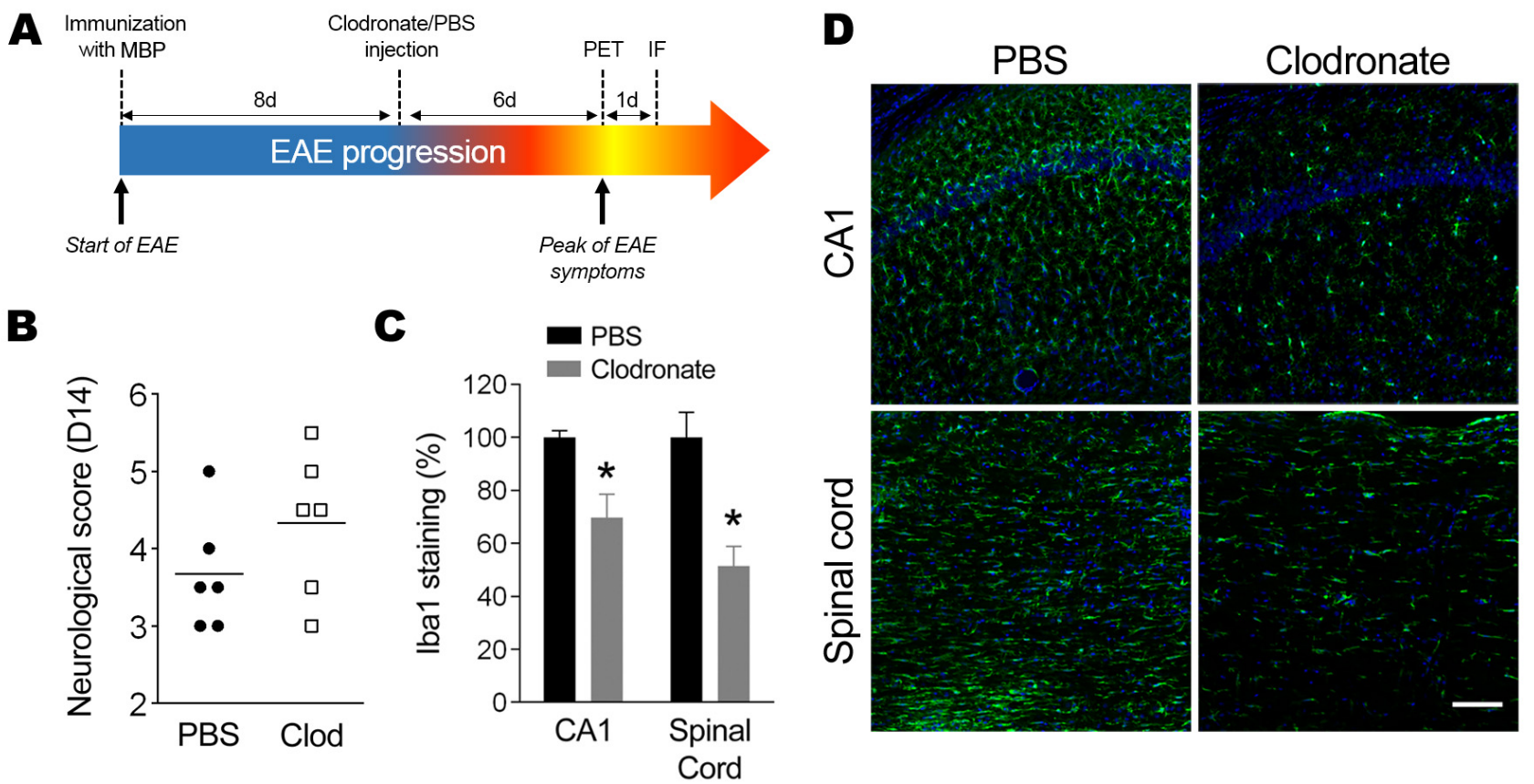


Figure 7.

$\left[{ }^{18} F\right] F S P G$
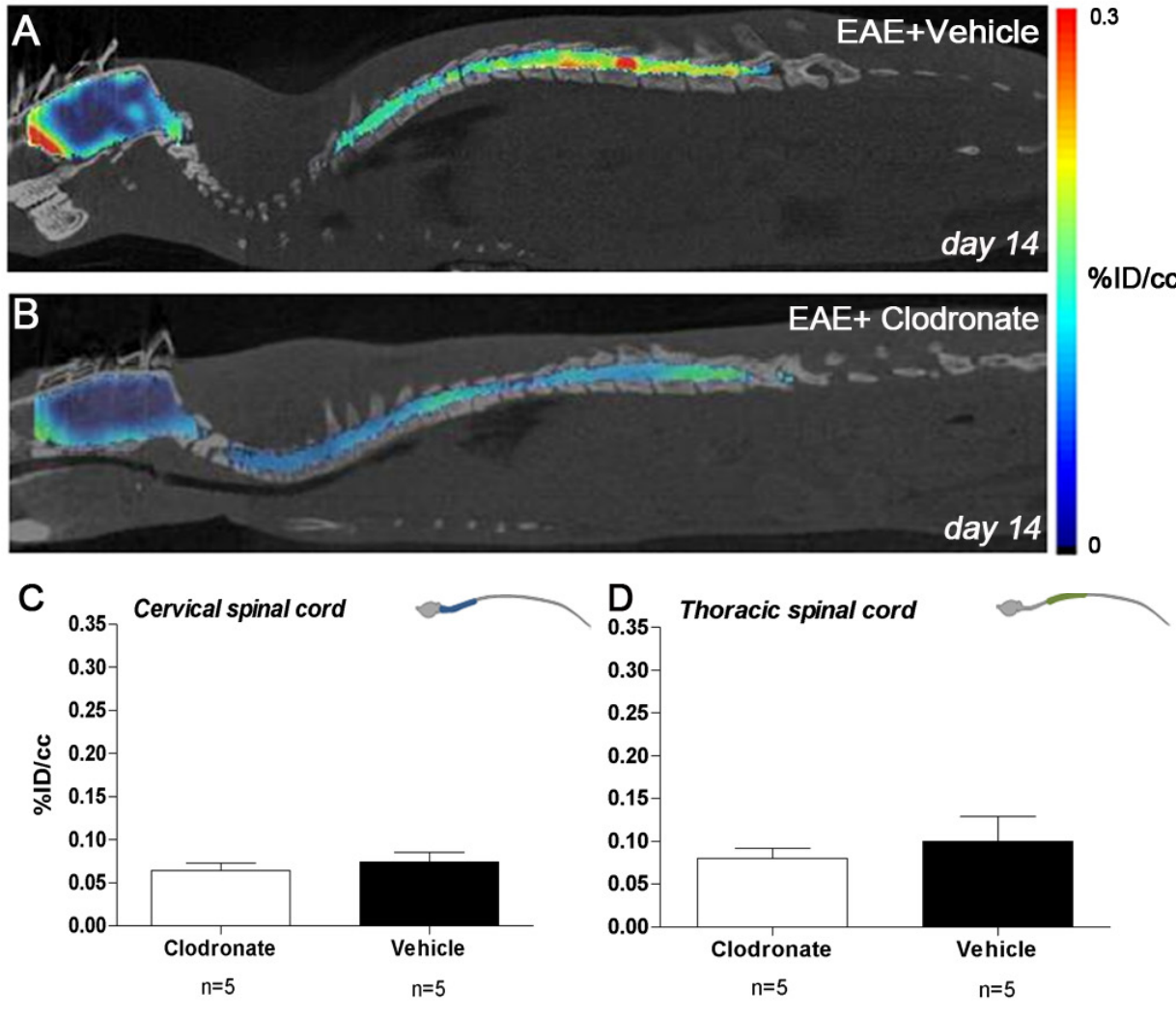

E

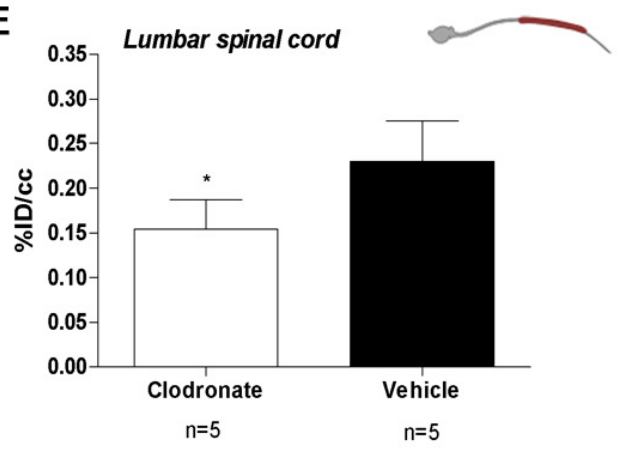

